\title{
Article \\ Grazing and Symmetry-Breaking Bifurcations Induced Oscillations in a Switched System Composed of Duffing and van der Pol Oscillators
}

\author{
Chun Zhang *, Qiaoxia Tang and Zhixiang Wang
}

check for updates

Citation: Zhang, C.; Tang, Q.;

Wang, Z. Grazing and

Symmetry-Breaking Bifurcations Induced Oscillations in a Switched System Composed of Duffing and van der Pol Oscillators. Mathematics 2022, 10, 772. https://doi.org/ $10.3390 /$ math10050772

Academic Editors: Yonghui Xia and Youhua Qian

Received: 22 January 2022

Accepted: 25 February 2022

Published: 28 February 2022

Publisher's Note: MDPI stays neutral with regard to jurisdictional claims in published maps and institutional affiliations.

Copyright: (c) 2022 by the authors. Licensee MDPI, Basel, Switzerland. This article is an open access article distributed under the terms and conditions of the Creative Commons Attribution (CC BY) license (https:// creativecommons.org/licenses/by/ $4.0 /)$.
School of Mathematical Science, Huaiyin Normal University, Huai'an 223300, China; qxtang@hytc.edu.cn (Q.T.); wzqiang@hytc.edu.cn (Z.W.)

* Correspondence: czhang@hytc.edu.cn

\begin{abstract}
By introducing a switching scheme related to the state and time, a typical switched model alternating between a Duffing oscillator and van der Pol oscillator is established to explore the typical dynamical behaviors as well as the mechanism of the switched system. Shooting methods to locate the limit cycle and specify bifurcation sets are described by defining an appropriate Poincaré map. Different types of multiple-Focus/Cycle and single-Focus/Cycle period oscillations in the system can be observed. Symmetry-breaking, period-doubling, and grazing bifurcation curves are obtained in the plane of bifurcation parameters, dividing the parameters plane into several regions corresponding to different kinds of oscillations. Meanwhile, based on the numerical simulation and bifurcation analysis, the mechanisms of several typical dynamical behaviors observed in different regions are presented.
\end{abstract}

Keywords: switched system; mixed-mode oscillation; nonsmooth evolution; bifurcation mechanism; transformed phase portrait

\section{Introduction}

Switched dynamic systems, which are an important class of nonsmooth systems, are described by the interplay between two or more continuous- or discrete-time subsystems, with a switching rule that is defined according to the critical values of the state variable or depends on the fixed time [1,2]. The main motivation to study a switched system is based on the fact that many natural systems, such as biological systems [3], manipulator robots [4], power electronics [5], and automobiles and locomotives with different gears [6] use switches and can be described in terms of switched systems. Meanwhile, the behavior of a switched system not only involves each subsystem but also is related to the switching scheme. Due to the fact that a switching scheme is inherently nonlinear, the whole system may lead to complex dynamic behaviors, such as periodic switching oscillations or even chaotic oscillations, etc. [7-9]. Since the existence of switches is widespread, and many interesting dynamical phenomena are found in switching systems, several scholars have focused on the switched system, reporting many results in recent years [10-13].

At present, the research into switched systems mainly focuses on stability analysis and control analysis. There are two basic problems in the study of the stability of a switched system. One is to study the stability of the system under an arbitrary switched scheme, based on the common Lyapunov function method. For example, Zhang [14] presented a recursive searching method of the common Lyapunov function (CLF) for the robust stable matrix set, based on which a sliding mode controller was designed, so that the system state remained on the sliding mode surface from the initial time instant. Zhu et al. [15] analyzed the issues with the stability of linear time-invariant switched systems with stable/unstable multiple equilibrium points and studied the design of a global asymptotic region-stabilzing controller. Kernani et al. [16] studied pole assignment stabilization with memory state 
feedback controllers for switched nonlinear time-varying delay systems based on novel common Lyapunov functions (CLFs), aggregation techniques, proprieties, and the BorneGentina criterion. The other is to research the stability of the system under a given switching scheme based on the multi-Lyapunov function method and average dwell time method. For example, Xie et al. [17] analyzed passivity and passive control for a cascade nonlinear system consisting of two switched systems and developed a asymptotic stability criterion for the cascade system without requiring the passivity of subsystem in the whole state interval. Liao et al. [18] investigated a class of nonlinear impulsive multidimensional switched systems and studied the passivity conditions of such systems by adopting the transition-dependent average dwell time and multiple Lyapunov functions. Kivilcim et al. [19] presented sufficient conditions for the near global stability of nonlinear switched systems consisting of both stable and unstable subsystems by the techniques from the stability analysis of the switched systems combined with the multiple Lyapunov density approach. In control analysis, how to control systems to achieve the stabilization control of the system or to find the optimal control of the appropriate switching control strategy is theoretically a very attractive task in switched systems. For example, from composite multiple Lyapunov-like functions and intermittently checking the direction of the worst state, Li et al. [20] designed a new intermittent compensation switching scheme to stabilize the switching nonlinear system. Bezborodov et al. [21] studied the issue of designing a switching scheme, which may ensure the stability of planar linear switched systems when the time delay affects the switching instants and proposed a switching table construction method based on minimum convergence speed.

Up to now, much of the previous literature has focused on the stability and controllability analysis or design of the switching conditions with different requirements. However, many problems such as dynamical behaviors, bifurcations associated with the switching conditions, and the mechanism of complexity with the variation in the parameters are seldom researched. Switched systems introduce many new characteristics, especially strong nonlinearity and singularity caused by the non-differentiability or discontinuity of vector fields $[22,23]$. Therefore, many dynamic characteristics of nonsmooth systems can not be treated by the ordinary smooth dynamic system theory, and special theories and methods need to be developed.

Duffing and van der Pol oscillators, which the simple nonlinear systems, may exhibit typical dynamical behaviors such as multiple states and self-excited oscillations. In order to study the complex dynamical characteristics as well as the evolution mechanism of the system with switching rules, a mathematical model alternating between Duffing and van der Pol oscillators with certain switch conditions related to the state variable and the fixed time is considered. Then, according to the property of the Poincare map of the periodic orbit and the tangency of the trajectory of the vector field and the switching surface, critical boundaries related to different forms of bifurcations are obtained. Subsequently, based on numerical simulation and bifurcation analysis the two parameter bifurcation sets of the switched system and the mechanisms of several typical dynamical behaviors observed in different regions are presented.

\section{Mathematical Model and Method}

\subsection{Hybrid System}

The autonomous van der Pol system, called subsystem $S_{1}$, can be described as

$$
S_{1}: \quad \ddot{x}-\beta\left(1-x^{2}\right) \dot{x}+x=0 .
$$

The stable limit cycle, $L C_{R}$, can be obtained, which indicates periodic vibration occurs in the vector field. Another system called the Duffing oscillator, expressed as $S_{2}$, can be written in the form

$$
S_{2}: \quad \ddot{x}+\delta \dot{x}-\alpha x+x^{3}=0,
$$


which may contain two stable focuses $E_{ \pm}=( \pm \sqrt{\alpha}, 0)$ and a saddle $E_{0}=(0,0)$ as $\delta>$ $0, \alpha>0$ on the condition $\sqrt{\delta^{2}-8 \alpha}>0$. When switches are introduced between the two subsystems, it may lead to movement alternating among the different types of behaviors. Here, the switching rules, which are defined according to the combination of the state variable and the fixed time are considered. Suppose that the switched system begins to be controlled by subsystem $S_{1}$ from an initial point $X_{0}$, when the trajectory in continuous state space hits the following local surface

$$
\Sigma_{1}=\left\{(x, y, t) \in R^{2} \times R^{+} \mid q(X)=x-x_{r e f}=0\right\},
$$

at $t=\tau_{1}$, the switched system will be redefined and controlled by subsystem $S_{2}$ for a fixed period of $T$, where $x_{r e f}$ is the fixed value related to the state. After a period of time $T+\tau_{1}$, the switched system may revert to subsystem $S_{1}$ until the state variable $x$ hits the surface $\Sigma_{1}$ again, and the motion then continues as above, where $y=\dot{x}$. Since the whole system alternates between subsystem $S_{1}$ and $S_{2}$, another local surface can be defined, expressed by

$$
\Sigma_{2}=\bigcup_{i=1}^{\infty}\left\{(x, y, t) \in R^{2} \times R^{+} \mid t=i T+\sum_{j=0}^{i+1} \tau_{j}\right\},
$$

where $\tau_{0}=0$ and $\left(\tau_{j}: j=1,2, \cdots\right)$ represent each time that the switch system runs in subsystem $S_{1}$. Therefore, the system with the switching schemes described above can be written as follows

$$
\begin{gathered}
\left\{\begin{array}{l}
\dot{x}=y \equiv f_{1}, \\
\dot{y}=-\mu x-\sigma\left(x^{2}-1\right) y \equiv g_{1},
\end{array} \quad t \in\left[i T+\sum_{j=0}^{i} \tau_{j}, i T+\sum_{j=0}^{i+1} \tau_{j}\right],\right. \\
\left\{\begin{array}{ll}
\dot{x} & =y \equiv f_{2}, \\
\dot{y} & =\alpha x-x^{3}-\delta y \equiv g_{2},
\end{array} \quad t \in\left[i T+\sum_{j=0}^{i+1} \tau_{j},(i+1) T+\sum_{j=0}^{i+1} \tau_{j}\right],\right.
\end{gathered}
$$

while the trajectory of the switched system is split into two parts: one is governed by subsystem $S_{1}$, denoted by

$$
X(t)=\Psi\left(t, X_{2 i}\right)=\left(\psi_{1}\left(t,\left(x_{2 i}, y_{2 i}\right)\right), \psi_{2}\left(t,\left(x_{2 i}, y_{2 i}\right)\right)\right) ;
$$

the other is determined by the behaviors of subsystem $S_{2}$, i.e.,

$$
X(t)=\Phi\left(t, X_{2 i+1}\right)=\left(\phi_{1}\left(t,\left(x_{2 i+1}, y_{2 i+1}\right)\right), \phi_{2}\left(t,\left(x_{2 i+1}, y_{2 i+1}\right)\right)\right),
$$

where $X_{2 i}, X_{2 i+1}, i=0,1,2, \cdots$ are the starting points of the two subsystems, respectively. Obviously, points $X_{2 i}, i=0,1,2, \cdots$ are on the local surface $\Sigma_{1}$, while points $X_{2 i+1}, i=$ $0,1,2, \cdots$ are on the local surface $\Sigma_{2}$.

\subsection{Limit Cycle and Poincaré Map}

In order to locate the limit cycle and formulate the conditions for its bifurcation, we first define a Poincaré map. We consider the situation, where the trajectory of the whole system exhibits nearly periodic motion with repeating transitions between subsystems $S_{1}$ and $S_{2}$ alternately. Figure 1 is a schematic illustration that shows part of the trajectory crossing the two local surfaces $\Sigma_{1}$ and $\sum_{2}$, alternately. Local maps from one section to the other one are defined as follows (see Figure 1)

$$
\begin{aligned}
P_{1}: \sum_{1} & \longrightarrow \sum_{2} \\
X_{0} & \longmapsto X_{1}=\Phi\left(T, X_{0}\right) \\
P_{2}: \sum_{2} & \longrightarrow \sum_{1} \\
X_{1} & \longmapsto X_{2}=\Psi\left(\tau_{1}, X_{1}\right)=\Psi\left(\tau_{1}, \Phi\left(T, X_{0}\right)\right) .
\end{aligned}
$$




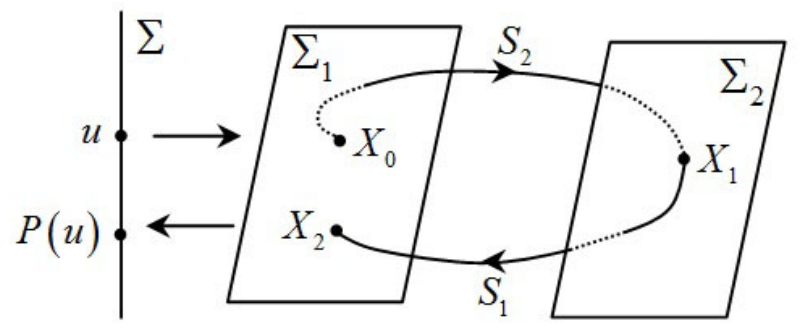

Figure 1. Schematic illustration for construction of the Poincaré map $P$ from the local surface and local maps.

It should noted here that the global coordinate $X_{0} \in \Sigma_{1} \subset \mathbb{R}^{2}$ is redundant to identify any point on the local surfaces. Thus, we define a local coordinate $\mathbf{u} \in \sum=\{(x, y) \mid x=$ $\left.x_{r e f}, y=\mathbf{u}\right\}$ and choose the projection $h$ and its inverse projection $h^{-1}$ as follows

$$
\begin{aligned}
h: \sum_{1} & \longrightarrow \sum_{\prime}\left(x_{r e f}, y, t\right) \longmapsto \mathbf{u}, \\
h^{-1}: \sum & \longrightarrow \sum_{1}, \mathbf{u} \longmapsto\left(x_{r e f}, y, t\right) .
\end{aligned}
$$

By treating $\sum$ as a Poincaré section, the Poincaré map $P$ can be defined as

$$
\begin{aligned}
P: \Sigma & \longrightarrow \sum \\
\mathbf{u} & \longmapsto h \circ \Psi \circ \Phi \circ h^{-1}(\mathbf{u})=h\left(\Psi\left(\tau_{1}, \Phi\left(T, h^{-1}(\mathbf{u})\right)\right)\right) .
\end{aligned}
$$

Similarly, a Poincaré map that indicates a limit cycle crossing the Poincaré map section $\mathrm{m}$ times is expressed by $P^{m}=h \circ(\Psi \circ \Phi)^{m} \circ h^{-1}$.

\subsection{Stability and Local Bifurcation}

A limit cycle of the whole system can be located by computing the related fixed point of the Poincaré map $P$, and the fixed point satisfies the following equation

$$
F_{l c}(\mathbf{u}) \equiv P(\mathbf{u})-\mathbf{u}=0 .
$$

In general, the exact analytic expression of the Poincaré map $P$ is unknown as is usual for a nonlinear system. The Jacobian matrix of $D P$ required for numerical computation is given by

$$
D P=\left(\frac{\partial \psi_{2}}{\partial x_{2 i}}-\frac{g_{1}}{f_{1}} \frac{\partial \psi_{1}}{\partial x_{2 i}}\right) \frac{\partial \phi_{1}}{\partial y_{2 i-1}}+\left(\frac{\partial \psi_{2}}{\partial y_{2 i}}-\frac{g_{1}}{f_{1}} \frac{\partial \psi_{1}}{\partial y_{2 i}}\right) \frac{\partial \phi_{2}}{\partial y_{2 i-1}} .
$$

The fixed point in (10) can be numerically solved by using numerical methods, and its stability can be determined by the following characteristic equation

$$
\chi(\mu) \equiv \operatorname{det}\left(\frac{\partial P}{\partial u}-\mu I_{n-1}\right)=0 .
$$

If all the exponents are located inside the unit circle on the complex plane, then the fixed point is stable. Furthermore, if one of the exponents crosses the unit circle outward from the inside by changing the parameters, the fixed point may become unstable corresponding to a codim-1 local bifurcation.

To specify a limit cycle, the position and its related local bifurcation can be obtained by the conditions as follows

$$
\mathbf{F}_{l b}(\mathbf{u}, \alpha)=\left[\begin{array}{c}
P(\mathbf{u})-\mathbf{u} \\
\chi(\mu)
\end{array}\right]=\mathbf{0}
$$

where $\alpha$ is a bifurcation parameter. For the fixed periodic solution of the switched system, saddle-node or picthfork bifurcation may occur when $\mu=1$, period-doubling bifurcation may take place once $\mu=-1$, while Neimark-Sacker bifurcation may happen once $\mu=e^{i \theta}$. 
These equations are simultaneously solved by Newton's method. The Jacobian matrix of $F_{l b}$ can be written as follows

$$
D \mathbf{F}_{l b}(\mathbf{u}, \alpha)=\left[\begin{array}{ll}
\frac{\partial P}{\partial \mathbf{u}}-I_{n-1} & \frac{\partial P}{\partial \alpha} \\
\frac{\partial \chi(\mu)}{\partial \mathbf{u}} & \frac{\partial \chi(\mu)}{\partial \alpha}
\end{array}\right] .
$$

\subsection{Grazing Bifurcation}

When a trajectory is tangent to the surface $\sum_{1}$, as illustrated in Figure 2, a slight change in a parameter value may cause the trajectory to intersect with the local surface and induce a change in dynamics. This critical change is called grazing bifurcation. The kind of contact of the smooth vector fields $F_{1}=\left(f_{1}, g_{1}\right)$ and $F_{2}=\left(f_{2}, g_{2}\right)$ with $\sum_{1}$ are provided by the directional Lie derivatives

$$
L_{F_{i}} q=\left\langle\nabla q, F_{i}\right\rangle=y \quad i=1,2
$$

where $\nabla q$ and $\langle\cdot, \cdot\rangle$ denote the gradient of the smooth function $q$ and the canonical inner product, respectively. On $\sum_{1}$ we distinguish the following crossing regions, defined by

$$
\begin{aligned}
& \sum_{1}^{+}=\left\{(x, y, t) \in \Sigma_{1} \mid x=x_{r e f}, y>0\right\}, \\
& \Sigma_{1}^{-}=\left\{(x, y, t) \in \Sigma_{1} \mid x=x_{r e f}, y<0\right\},
\end{aligned}
$$

which implies that the grazing point on a trajectory is only possible on the boundary region

$$
\Sigma_{1}^{0}=\left\{(x, y, t) \in \Sigma_{1} \mid x=x_{r e f}, y=0\right\} .
$$

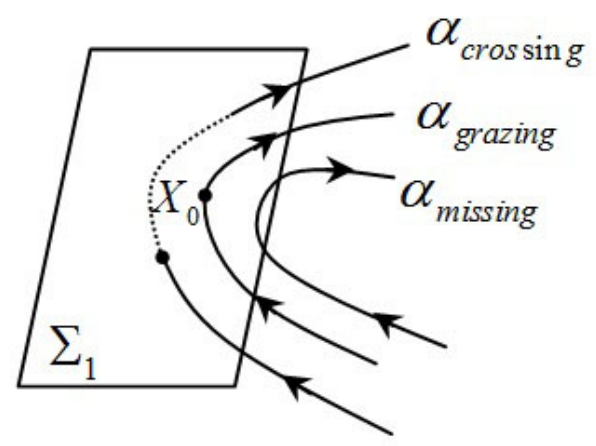

Figure 2. Schematic illustration for grazing trajectory tangent to section $\sum_{1}$ at $X_{0}$. A trajectory crosses the section or does not touch the section $\sum_{1}$ when $\alpha_{\text {grazing }}$ is increased or decreased.

Next, we determine the conditions of the periodic grazing solution by considering the return time of a limit cycle as an unknown variable. Suppose that $X_{0}$ on $\sum_{1}$ is the initial point of a grazing trajectory, and the trajectory transversally intersects $\sum_{1}(2 m-1)$-times alternately; then, finally it becomes tangent to the local surface $\Sigma_{1}$. For convenience, we define the following maps

$$
\begin{aligned}
\bar{P}_{2 k-1}: \sum_{1} & \longrightarrow \sum_{1} \\
X_{0} & \longmapsto X_{2 k-1}=P_{1} \circ\left(P_{2} \circ P_{1}\right)^{k-1}\left(X_{0}\right) \\
\bar{P}_{2 k}: \sum_{1} & \longrightarrow \sum_{1} \\
X_{0} & \longmapsto X_{2 k}=\left(P_{2} \circ P_{1}\right)^{k}\left(X_{0}\right),
\end{aligned}
$$

where $k=1,2, \cdots, m$. Obviously, based on the switching scheme, the traveling time from $X_{2 k}$ to $X_{2 k+1}$ is a fixed time $T$, while the traveling time from $X_{2 k+1}$ to $X_{2 k+2}$ is denoted by $\tau_{k+1}\left(X_{2 k+1}\right)$ for $k=0,1,2, \cdots, m-1$. Then, the cumulative traveling time from $X_{0}$ to $X_{2 m}$ is denoted by

$$
\bar{\tau}_{2 k}\left(X_{0}\right)=\bar{\tau}_{2 k-1}\left(X_{0}\right)+\tau_{k}\left(X_{2 k-1}\right),
$$




$$
\bar{\tau}_{2 k-1}\left(X_{0}\right)=\bar{\tau}_{2 k-2}\left(X_{0}\right)+T,
$$

where $k=1,2, \cdots, m, \bar{\tau}_{0}\left(X_{0}\right) \equiv 0$. The above setting provides a map dependent on the total time $\tau$ and the initial condition $X_{0}$ as follows

$$
\bar{P}\left(\tau, X_{0}\right)=\Psi\left(\tau-\bar{\tau}_{2 m-1}\left(X_{0}\right), \Phi\left(T, X_{2 m-2}\right)\right),
$$

where $\tau>\bar{\tau}_{2 m-1}\left(X_{0}\right)$. Upon the map $\bar{P}$, the condition of a grazing bifurcation of an $m$-fold periodic oscillation can be formulated as follows

$$
\mathbf{F}_{g b}\left(X_{0}, \tau, \alpha\right)=\left[\begin{array}{c}
\bar{P}\left(\tau, X_{0}\right)-X_{0} \\
q\left(\bar{P}\left(\tau, X_{0}\right)\right) \\
\frac{\partial q}{\partial X} \cdot F_{2}\left(\bar{P}\left(\tau, X_{0}\right)\right)
\end{array}\right]=\mathbf{0} .
$$

The first equation indicates that a trajectory starting from $X_{0}$ on $\Sigma_{1}$ may return back to $X_{0}$ leading to an $m$-folded limit cycle with period $\tau$. The second equation represents that the trajectory is located on $\Sigma_{1}$ at time $\tau$. The third equation means that the trajectory is tangent to the surface $\Sigma_{1}$ at time $\tau$, where $\alpha$ is a bifurcation parameter.

\section{Results}

In this section, based on the numerical method introduced in the previous section, we fix some of the parameters at $\beta=1.0, \delta=1.0$, and $T=4.5$, and take parameters $\lambda$ and $x_{\text {ref }}$ as bifurcation to investigate the dynamical evolution of the oscillator. Since different initial conditions may influence the structure of the attractors of the switched system, all the tests presented in this paper have been performed for the initial condition $\left(x_{0}, y_{0}\right)=(0.5,0.1)$.

Figure 3 shows a bifurcation diagram of the limit cycles in the $\left(x_{r e f}, \lambda\right)$ plane, indicating the parameter region of no switching together with the bifurcation curves of limit cycles. Both side regions correspond to no switching, where the whole system is only governed by subsystem $S_{1}$.

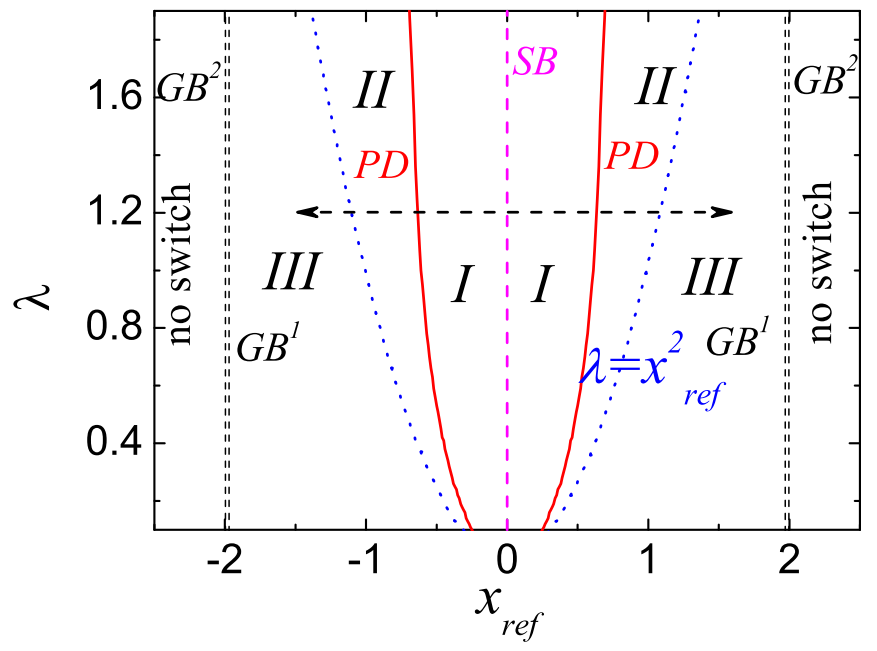

Figure 3. Bifurcation diagram of the limit cycles in the $\left(x_{r e f}, \lambda\right)$ plane. Period-doubling, symmetrybreaking, and grazing bifurcations of the limit cycle are denoted by $P D, S B$, and $G B$, respectively.

Let us examine in detail the oscillation and bifurcation mechanisms of the limit cycle. The period 2 oscillation exists in the region I. For example, when $x_{r e f}=0.53, \lambda=1.2$, the fixed points and the exponent can be computed at $\mathbf{u}_{1}=(0.53,-1.007) \in \Sigma_{1}^{-}$, $\mathbf{u}_{2}=$ $(0.53,2.003) \in \sum_{1}^{+}$and $\mu=0.673$, respectively, indicating that a stable period 2 trajectory crossing the surface $\sum_{1}$ twice can be observed (see in Figure 4a the light gray trajectory). When $x_{\text {ref }}=-0.53, \lambda=1.2$, another stable period 2 trajectory (see in Figure 4a the blue trajectory) can be obtained, the fixed points of which are $\mathbf{u}_{1}=(-0.53,1.007) \in \Sigma_{1}^{-}$and 
$\mathbf{u}_{2}=(-0.53,-2.003) \in \sum_{1}^{+}$. It can be seen that the two stable period 2 trajectories are center symmetric around the origin of the coordinates, which implies that when the parameters are taken as axially symmetric around the line $x_{r e f}=0.0$, the periodic oscillations may center symmetrically around the origin of the coordinates. Meanwhile, based on the dynamics of the subsystems, one can see that the vector fields of the whole system may alternate between the transient process of the stable focuses $E_{ \pm}$and the stable limit cycle $L C_{R}$, forming the multiple-Focus/Cycle period 2 oscillation in region $I$.

When the parameters change from region $I$ to the line $x_{r e f}=0.0$, the period 2 oscillations on both sides of the line $x_{r e f}=0.0$ may collide with each other and suddenly change to an unstable period oscillation, which is also a multiple-Focus/Cycle oscillation, but it is center symmetric around the origin of coordinates with itself, via symmetry breaking bifurcation. The typical unstable symmetric multiple-Focus/cycle period 2 trajectory is presented in Figure $4 b$.
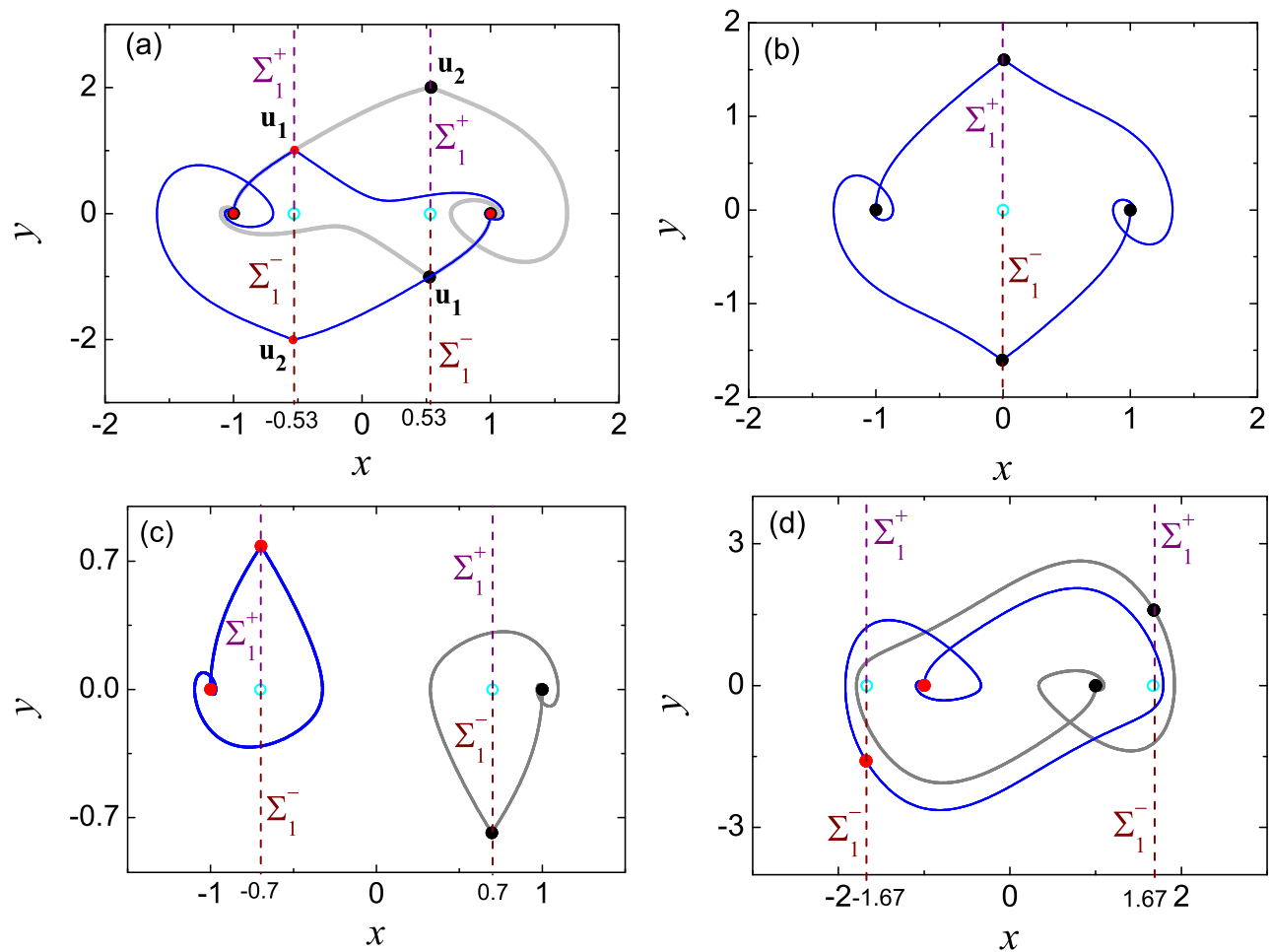

Figure 4. (a,b) Typical multiple-Focus/Cycle period 2 oscillations and (c,d) typical single-Focus/Cycle period 1 oscillations for $\lambda=1.2$. (a) $x_{r e f}= \pm 0.53$; (b) $x_{r e f}=0.0$; (c) $x_{r e f}= \pm 0.7$; (d) $x_{r e f}= \pm 1.67$.

Crossing the bifurcation curve of $P D$ from the region $I$ to $I I$, the multiple-Focus/Cycle period 2 oscillation may become unstable at $P D$, leading to a stable period 1 oscillation via reversed period-doubling bifurcation. The typical phase portraits in region $I I$ are presented in Figure 4c. As shown in Figure 4c, it can be seen that the trajectory of blue period 1 oscillation may alternate between the transient process of the stable focus $E_{-}$and the stable limit cycle $L C_{D}$, while the gray period 1 oscillation may alternate between the transient process of another stable focus $E_{+}$and the stable limit cycle $L C_{D}$. These type of oscillations are called single-Focus/Cycle period 1 oscillations.

In region $I I I$, another single-Focus/Cycle period 1 oscillation is obtained, as shown in Figure $4 d$, from which we find that the structure of the periodic switching may differ from the period 1 oscillations in region $I I$, although the switching attractors still alternate between stable focus $E_{-}$and stable limit cycle $L C_{R}$ or stable focus $E_{+}$and stable limit cycle $L C_{R}$. The phenomenon can be understood by analyzing the change in the location of the fixed point between the crossing regions $\Sigma_{1}^{-}$and $\Sigma_{1}^{+}$. For $\lambda=1.2$ and $x_{\text {ref }}$ decreasing from -1.09 to -1.0954 , the switched system behaves as a single-Focus/Cycle period 1 oscillation 
with the fixed point on $\sum_{1}^{+}$(typical phase diagram see Figure $4 \mathrm{c}$, the blue trajectory). However, when $x_{\text {ref }}$ decreases through $-\sqrt{\lambda}=-1.0954$, the fixed point suddenly jumps to the crossing region $\sum_{1}^{-}$(see Table 1), resulting another type of stable single-Focus/Cycle period 1 oscillation (see Figure $4 \mathrm{~d}$, the blue trajectory). When $x_{r e f}$ increases through $\sqrt{\lambda}=1.0954$, the fixed point suddenly jumps to the crossing region $\sum_{1}^{+}$from $\Sigma_{1}^{-}$(see Table 1), the period 1 oscillation (see Figure $4 \mathrm{c}$, light gray trajectory) may change to another period 1 oscillation (see Figure $5 d$, light gray trajectory). Based on the discussion, it can be seen that these two types of single-Focus/Cycle period 1 oscillations may be divided by the curve $\lambda=x_{r e f}^{2}$ (see in Figure 3, the parameter in which one of the stable focuses $E_{ \pm}$ happens to be on the surface $\sum_{1}$ ).

Table 1. The situation of the fixed point on the crossing regions for $\lambda=1.2$ and different $x_{r e f}$.

\begin{tabular}{cccccc}
\hline$x_{\text {ref }}$ & Fixed Point & Crossing Region & $x_{\text {ref }}$ & Fixed Point & Crossing Region \\
\hline-1.09 & $(-1.09,0.10821)$ & $\sum_{1}^{+}$ & 1.09 & $(1.09,-0.10821)$ & $\sum_{1}^{-}$ \\
-1.0948 & $(-1.0948,0.0436)$ & $\sum_{1}^{+}$ & 1.0948 & $(1.0948,-0.0436)$ & $\sum_{1}^{-}$ \\
-1.0952 & $(-1.0952,0.0219)$ & $\sum_{1}^{+}$ & 1.0952 & $(1.0952,-0.0219)$ & $\sum_{1}^{-}$ \\
-1.0954 & $(-1.0954,0.0109)$ & $\sum_{1}^{+}$ & 1.0954 & $(1.0954,-0.0109)$ & $\sum_{1}^{-}$ \\
-1.0955 & $(-1.0955,-2.5560)$ & $\sum_{1}^{-}$ & 1.0955 & $(1.0955,2.5560)$ & $\sum_{1}^{+}$ \\
-1.1 & $(-1.1,-2.5384)$ & $\sum_{1}^{-}$ & 1.1 & $(1.1,2.5384)$ & $\sum_{1}^{+}$ \\
\hline
\end{tabular}

When $x_{\text {ref }}$ decreases along the horizontal arrowed line $\alpha=1.2$, the fixed point on the crossing region $\Sigma_{1}^{-}$(see Figure 5a fixed point $\mathbf{u}^{*}$ ) may gradually reach the boundary region $\sum_{1}^{0}$ at $x_{\text {ref }}=-1.98797$ (see Table 2), which implies that the single-Focus/Cycle period 1 oscillation may become unstable at $x_{\text {ref }}=-1.98797$, resulting a unstable period 1 oscillation via grazing bifurcation $G B^{1}$ (see Figure 3). A typical unstable single-Focus/Cycle period 1 grazing oscillation is shown in Figure $5 b$ (the blue trajectory). By further increasing $x_{\text {ref }}$, the fixed point on $\sum_{1}^{0}$ will return back to the crossing region $\Sigma_{1}^{-}$(see Figure $5 \mathrm{c}$, fixed point $\mathbf{u}^{*}$ ) and then reach $\sum_{1}^{0}$ again at $x_{r e f}=-1.9958$ (see Table 2), indicating grazing bifurcation $G B^{2}$ (see Figure 3) takes place at $x_{r e f}=-1.9958$. Another typical unstable singleFocus/Cycle period 1 grazing oscillation is obtained in Figure $5 \mathrm{~d}$. When $x_{\text {ref }}$ increases along the horizontal arrowed line $\alpha=1.2$, the unstable single-Focus/Cycle period 1 grazing oscillations (the light grey trajectories in Figure $5 b, d$ ), which are center symmetric around the origin of the coordinates with the blue grazing trajectories in Figure $5 \mathrm{~b}$,d, respectively, are obtained. The mechanism of bifurcation can also be explored through the analysis of the change in the location of the fixed point between the different regions, seen in Table 2.

Table 2. The situation of the fixed point on the crossing regions for $\lambda=1.2$ and different $x_{\text {ref }}$.

\begin{tabular}{cccccc}
\hline$x_{\text {ref }}$ & Fixed Point & Crossing Region & $x_{\text {ref }}$ & Fixed Point & Crossing Region \\
\hline-1.9871 & $(-1.9871,-0.0598)$ & $\sum_{1}^{-}$ & 1.9871 & $(1.9871,0.0598)$ & $\sum_{1}^{+}$ \\
-1.9877 & $(-1.9877,-0.0388)$ & $\sum_{1}^{-}$ & 1.9877 & $(1.9877,0.0388)$ & $\sum_{1}^{+}$ \\
-1.9879 & $(-1.9879,-0.00182)$ & $\sum_{1}^{-}$ & 1.9879 & $(1.9879,0.00182)$ & $\sum_{1}^{+}$ \\
-1.98797 & $(-1.98797,0.0)$ & $\sum_{1}^{0}$ & 1.98797 & $(1.98797,0.0)$ & $\sum_{1}^{0}$ \\
-1.988 & $(-1.988,-0.1770)$ & $\sum_{1}^{-}$ & 1.988 & $(1.988,0.1770)$ & $\sum_{1}^{+}$ \\
-1.995 & $(-1.995,-0.061)$ & $\sum_{1}^{-}$ & 1.995 & $(1.995,0.061)$ & $\sum_{1}^{+}$ \\
-1.9957 & $(-1.9957,-0.0191)$ & $\sum_{1}^{-}$ & 1.9957 & $(1.9957,0.0191)$ & $\sum_{1}^{+}$ \\
-1.9958 & $(-1.9958,0.0)$ & $\sum_{1}^{0}$ & 1.9958 & $(1.9958,0.0)$ & $\sum_{1}^{0}$ \\
\hline
\end{tabular}



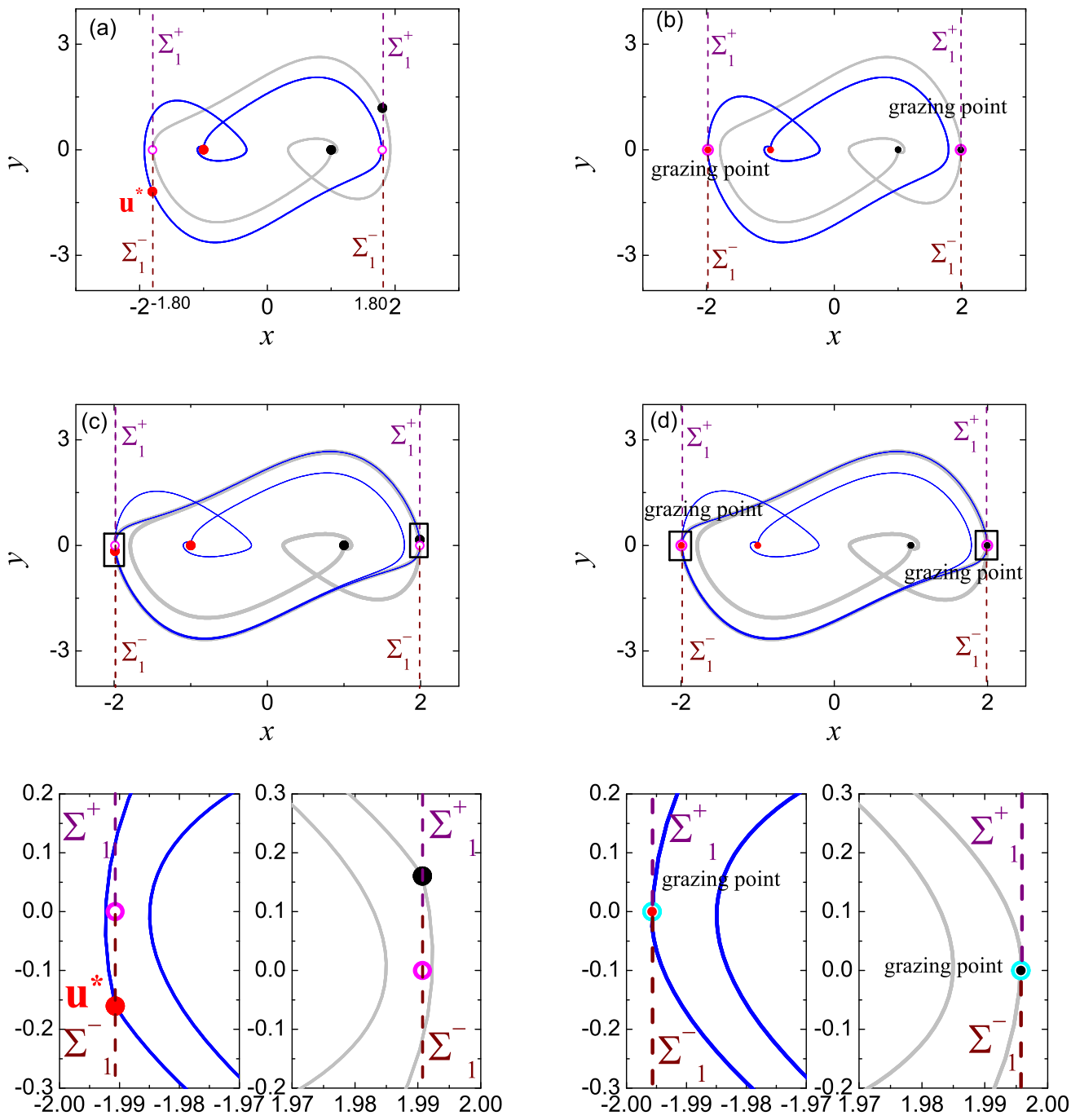

Figure 5. (a,c) two Two types of stable single-Focus/Cycle period 1 oscillations and (c,d) two types of unstable single-Focus/Cycle period 1 grazing oscillations for $\lambda=1.2$, where the subfigures give a clear understanding of the rectangular region. (a) $x_{r e f}= \pm 1.80$; (b) $x_{r e f}= \pm 1.9879$; (c) $x_{r e f}= \pm 1.99$; (d) $x_{r e f}= \pm 1.9958$.

\section{Conclusions}

Based on a switching scheme related to the state and time, a typical switched model alternating between a Duffing oscillator and a van der Pol oscillator was established, which exhibited complex combined movements, such as multiple-Focus/Cycle and singleFocus/Cycle periodic oscillations. The existence of these periodic oscillations were located by solving the fixed point of the Poincaré map. Conducting bifurcation analysis on the Poincaré map and considering the tangency of the trajectory of the vector field and the switching surface, two-parameter bifurcation diagrams were obtained. This study shows that symmetry-breaking bifurcation, period-doubling bifurcation, and grazing bifurcation play an important role in the transition from one type of regular oscillation to another oscillation. The results we obtained in this paper may help to understand different oscillation behaviors and the associated mechanisms of the switched system.

Author Contributions: Formal analysis, C.Z.; methodology, C.Z. and Z.W.; writing-original draft preparation, Q.T.; writing-review and editing, Q.T. All authors have read and agreed to the published version of the manuscript. 
Funding: This work was supported by the National Natural Science Foundation of China (Grant Nos. 11502091 and 11801209)

Institutional Review Board Statement: Not applicable.

Informed Consent Statement: Not applicable.

Data Availability Statement: Not applicable.

Acknowledgments: We will like to express our deep thanks to the anonymous referees for their valuable comments.

Conflicts of Interest: The authors declare no conflict of interest.

\section{References}

1. Goebel, R.; Sanfelice, R.G.; Teel, A.R. Invariance principles for switching systems via hybrid systems techniques. Syst. Control Lett. 2008, 57, 980-986. [CrossRef]

2. Zhang, C.; Yu, Y.; Han, X.J.; Bi, Q.S. Dynamical behaviors of a system with switches between the Rössler oscillator and Chua's circuits. Chin. Phys. B 2012, 21, 100501. [CrossRef]

3. Liefferinckx, C.; Cremer, A.; Franchimont, D. Switching biologics used in inflammatory bowel diseases: How to deal with in practice. Curr. Opin. Pharmacol. 2020, 55, 82-89. [CrossRef] [PubMed]

4. Kolur, K.; Chintalapudi, S.; Boots, B.; Mukadam, M. Online Motion Planning Over Multiple Homotopy Classes with Gaussian Process Inference. In Proceedings of the 2019 IEEE/RSJ International Conference on Intelligent Robots and Systems (IROS), Venetian Macao, Macau, 4-8 November 2019; pp. 2358-2364.

5. $\quad$ Feng, Z.X.; Sun, W.Q.; Zhu, J.; Shao, J.Y.; Hu, W.S. Resource Allocation in Electrical/Optical Hybrid Switching Data Center Networks. J. Opt. Commun. Netw. 2017, 9, 648-657. [CrossRef]

6. Aiso, K.; Akatsu, K.; Aoyama, Y. A Novel Flux Switching Magnetic Gear for High Speed Motor System. In Proceedings of the 44th Annual Conference of the IEEE Industrial-Electronics-Society (IECON), Washington, DC, USA, 21-23 October 2018; pp. 337-342.

7. Zhang, C.; Han, X.J.; Bi, Q.S. Dynamical behaviors of the periodic parameter-switching system. Nonlinear Dyn. 2013, 73, 29-37. [CrossRef]

8. Li, F.; Liu, Y.Y. Limit cycles in a class of switching system with a degenerate singular point. Chaos Solitons Fract. 2016, 92, 86-90. [CrossRef]

9. Wagner, V.; Radde, N.E. SiCaSMA: An Alternative Stochastic Description via Concatenation of Markov Processes for a Class of Catalytic Systems. Mathematics 2021, 9, 1074. [CrossRef]

10. Liu, S.; Pang, H.B. Finite-Time Incremental Passivity and Output Tracking Control for Switched Nonlinear Systems. IEEE Access 2020, 8, 168526-168533. [CrossRef]

11. Harrison, J.; Yeung, E.N. Stability Analysis of Parameter Varying Genetic Toggle Switches Using Koopman Operators. Mathematics 2021, 9, 3133. [CrossRef]

12. Liao, F.C.; Cui, L.J.; Lu, Y.R.; Deng, J.M. Preview Tracking Control of Linear Periodic Switched Systems with Dwell Time. Math Probl. Eng. 2020, 2020, 8395683. [CrossRef]

13. Makarenkov, O. A linear state feedback switching rule for global stabilization of switched nonlinear systems about a nonequilibrium point. Eur. J. Control 2019, 49, 62-67. [CrossRef]

14. Zhang, X.Y. Robust integral sliding mode control for uncertain switched systems under arbitrary switching rules. Nonlinear Anal. Hybrid Syst. 2020, 37, 100900. [CrossRef]

15. Zhu, L.Y.; Qiu, J.B. Region stability and stabilisation of switched linear systems with multiple equilibria. Int. J. Control. 2019, 92, 1061-1083. [CrossRef]

16. Kermani, M.; Sakly, A. Pole assignment stabilization for a class of switched nonlinear time-varying delay systems. ISA Trans. 2020, 106, 138-151. [CrossRef] [PubMed]

17. Xie, H.Z.; Yang, D.; Zong, G.D. Passivity and passive control for switched nonlinear systems based on multiple storage functions technique. Int. J. Control 2020, 95, 22-32. [CrossRef]

18. Liao, Z.R.; Wang, Y. Passivity Analysis of Nonlinear Impulsive Multi-Dimensional Switched Systems. In Proceedings of the 2021 American Control Conference, New Orleans, LA, USA, 25-28 May 2021; pp. 846-851.

19. Kivilcim, A.; Karabacak, O.; Wisniewski, R. Almost Global Stability of Nonlinear Switched System with Stable and Unstable Subsystems. In Proceedings of the 59th IEEE Conference on Decision and Control (CDC), Jeju, Korea, 14-18 December 2020; pp. 3285-3290.

20. Li, Z.J.; Ma, Y.J.; Zhao, J. Control Design of switched Nonlinear Systems: An Intermittend Compensation Switching Strategy. SIAM J. Control Optim 2020, 56, 3684-3708. [CrossRef]

21. Bezborodov, V.; Di Persio, L.; Muradore, R. Stabilization of planar non-Markovian switched linear systems with unbounded random delays. Eur. J. Control 2021, 57, 109-118. [CrossRef]

22. Zhang, C.; Han, X.J.; Bi, Q.S. On symmetry-breaking bifurcation in the periodic parameter-switching Lorenz oscillator. Sci. China Technol. Sci. 2013, 56, 2310-2316. [CrossRef]

23. Zhang, C.; Bi, Q.S.; Han, X.J.; Zhang, Z.D. On two-parameter bifurcation analysis of switched system composed of Duffing and van der Pol oscillators. Commun. Nonlinear Sci. Numer. Simul. 2014, 19, 750-757. [CrossRef] 